\title{
Increase in Sodium Conductance Decreases Firing Rate and Gain in Model Neurons
}

\author{
Tilman J. Kispersky, ${ }^{\star}$ Jonathan S. Caplan, ${ }^{\star}$ and Eve Marder \\ Biology Department and Volen Center, Brandeis University, Waltham, Massachusetts 02454-9110
}

\begin{abstract}
We studied the effects of increased sodium conductance on firing rate and gain in two populations of conductance-based, single-compartment model neurons. The first population consisted of 1000 model neurons with differing values of seven voltage-dependent conductances. In many of these models, increasing the sodium conductance threefold unexpectedly reduced the firing rate and divisively scaled the gain at high input current. In the second population, consisting of 1000 simplified model neurons, we found that enhanced sodium conductance changed the frequencycurrent (FI) curve in two computationally distinct ways, depending on the firing rate. In these models, increased sodium conductance produced a subtractive shift in the FI curve at low firing rates because the additional sodium conductance allowed the neuron to respond more strongly to equivalent input current. In contrast, at high input current, the increase in sodium conductance resulted in a divisive change in the gain because the increased conductance produced a proportionally larger activation of the delayed rectifier potassium conductance. The control and sodium-enhanced FI curves intersect at a point that delimits two regions in which the same biophysical manipulation produces two fundamentally different changes to the model neuron's computational properties. This suggests a potentially difficult problem for homeostatic regulation of intrinsic excitability.
\end{abstract}

\section{Introduction}

The electrical excitability of spiking neurons is often characterized by measuring the action potential frequency in response to injected current to generate a frequency-current (FI) curve. The slope of the FI curve is referred to as the gain of the neuron. Changes to the FI curve (Holt and Koch, 1997; Chance et al., 2002; Prescott and De Koninck, 2003) can be additive (a change in rheobase, the minimum current required to elicit spiking) or multiplicative (a change in FI curve slope). Multiplicative changes to the FI curve are of interest because they are thought necessary to implement complex behaviors such as coordinate transformations for reaching motions (Salinas and Thier, 2000) and detection of looming objects in the visual field (Gabbiani et al., 2002). Changes to the neuronal input-output relationship (gain control) are thought to be fundamental mechanisms by which neurons maintain appropriate response levels to the changing features of their synaptic inputs (Destexhe et al., 2003; Silver, 2010) and can alter the computational properties of neurons (Lundstrom et al., 2009; Silver, 2010).

The number and kind of voltage-dependent conductances in each neuron determine its excitability and the way it responds to external drive. Therefore, it is of interest to understand how the FI curves and gains of spiking neurons depend on the $\mathrm{Na}^{+}$conductance. The initial purpose of this study was to determine the

\footnotetext{
Received April 9, 2012; revised June 11, 2012; accepted June 15, 2012.

Author contributions: T.J.K. and E.M. designed research; T.J.K. and J.S.C. performed research; T.J.K. and J.S.C. analyzed data; T.J.K. and E.M. wrote the paper.

This work was supported by NIH Grants MH 46742 (T.J.K. and E.M.) and T90 DA032435 (J.S.C.). We thank Dr. Fernando R. Fernandez for helpful comments on the manuscript and Dr. Ted Brookings and Dr. Timothy O'Leary for useful discussions.

The authors declare no competing financial interests.

*T.J.K. and J.S.C. contributed equally to this work.

Correspondence should be addressed to Dr. Tilman J. Kispersky, Department of Biology, MS 013 Brandeis University, 415 South Street, Waltham, MA 02454-9110. E-mail: tilman@brandeis.edu.

DOI:10.1523/JNEUROSCI.2045-12.2012

Copyright $\odot 2012$ the authors $\quad 0270-6474 / 12 / 3210995-10 \$ 15.00 / 0$
}

effects of raised $\mathrm{Na}^{+}$conductance on neuronal gain. Because intrinsic conductance densities can be quite variable across neurons of the same cell type (Golowasch et al., 1999; Swensen and Bean, 2005; Schulz et al., 2006, 2007; Goaillard et al., 2009), we generated a set of models in which each individual model was constructed with random maximal conductances. This approach, to assess the robustness of a given phenomenon by probing parameter space in a randomized fashion, has been used before (Goldman et al., 2001; Prinz et al., 2004; Günay et al., 2008; Taylor et al., 2009; Marder, 2011; Marder and Taylor, 2011) and provides information about whether a model behavior is unique relative to the space of many similarly constructed models.

Our initial expectation was that increasing the $\mathrm{Na}^{+}$conductance would enhance excitability across a wide range of inputs. Instead, this study shows that, while excitability is enhanced at low current injection levels, the opposite often occurs with high current injection that results in high firing rates. Thus, increases in $\mathrm{Na}^{+}$conductance in response to decreased activity (Desai et al., 1999) would be homeostatic for conditions of low external drive, but might produce paradoxical anti-homeostatic changes at higher levels of external drive. These results suggest revisions of our thinking about what the targets of homeostatic regulation should be to ensure a neuron can stably regulate its gain.

\section{Materials and Methods}

Simulations. Simulations were run on a multicore personal computer using MATLAB (The MathWorks). The model used in the computational experiments was derived from a model that has been previously described in detail (Turrigiano et al., 1995; Liu et al., 1998). This version of the model removed all of the calcium-dependent homeostatic mechanisms leaving only static biophysical conductances. Our results are produced by running the model with a full set of conductances and a reduced version of the model in which many of the conductances were removed. The full-conductance model has seven active conductances as well as a leak conductance. These active chan- 
Table 1. Functions used to describe channel activation and inactivation kinetics

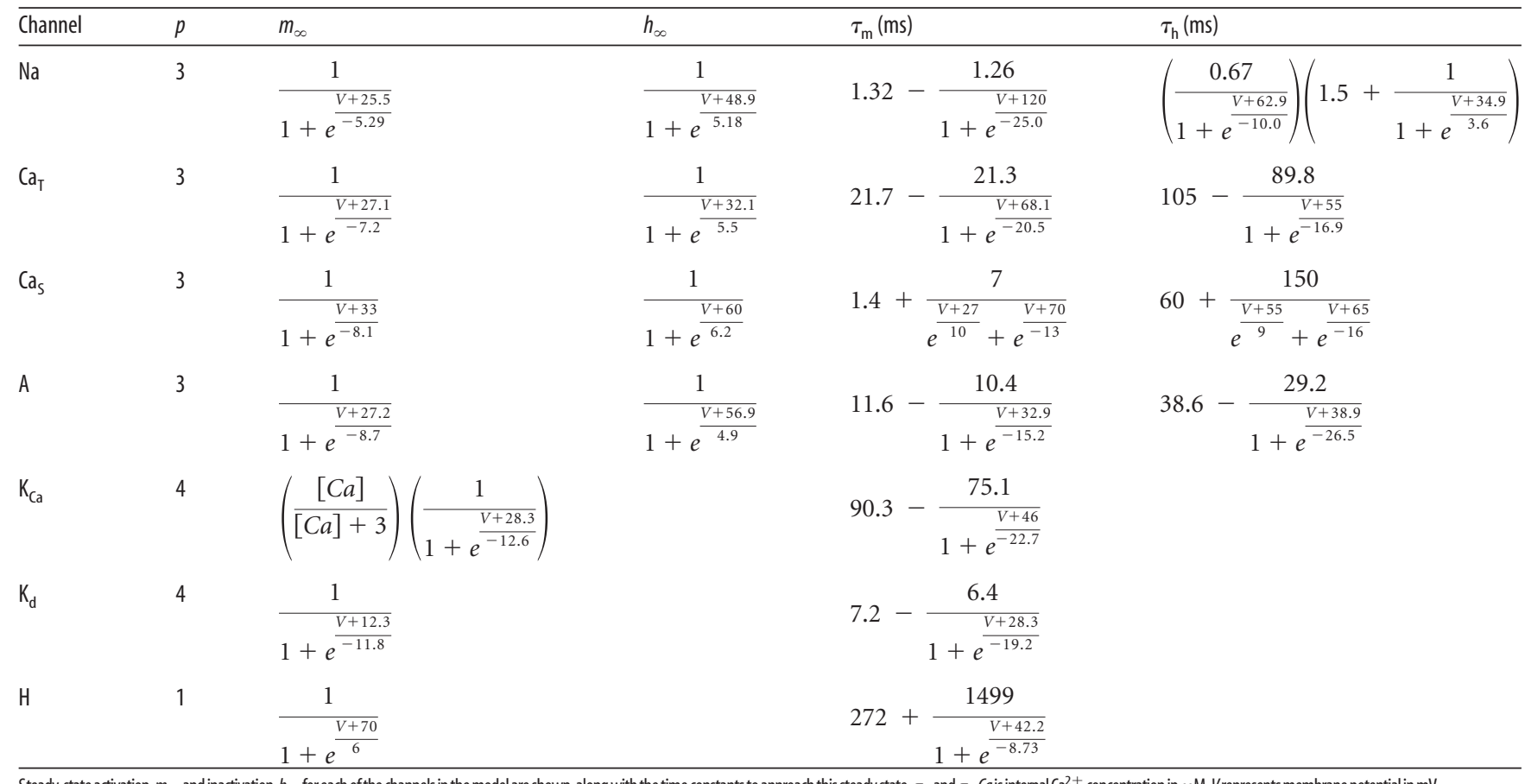

Steady-state activation, $m_{\infty}$ and inactivation, $h_{\infty,}$ for each of the channels in the model are shown, along with the time constants to approach this steady state, $\tau_{\mathrm{m}}$ and $\tau_{\mathrm{h}}$. Ca is internal Ca ${ }^{2+}$ concentration in $\mu \mathrm{M}$. $V$ represents membrane potential in $\mathrm{mV}$.

nels are $\mathrm{Na}^{+}$(fast sodium), $K_{\mathrm{d}}$ (delayed rectifier potassium), $K_{\mathrm{Ca}}$ (calciumgated potassium), $\mathrm{Ca}_{\mathrm{T}}^{2+}$ (transient calcium), $\mathrm{Ca}_{\mathrm{S}}^{2+}$ (slow calcium), A (potassium), and $H$ (hyperpolarization-activated mixed cation). The reduced model has only $\mathrm{Na}, K_{\mathrm{d}}, A$, and leak channels.

All currents besides calcium are calculated as

$$
I_{i}=\bar{g}_{i} m_{i}^{p} h_{i}\left(V_{\mathrm{m}}-E_{i}\right),
$$

where $i$ represents the individual channel type, $p$ is the power to which the activation variable is raised, $\bar{g}_{i}$ the maximal channel conductance, $m_{i}^{p}$ the activation state variable, and $h_{i}$ the inactivation state variable. $V_{\mathrm{m}}$ is membrane potential and $E_{i}$ is reversal potential for that channel type. The potassium reversal potential is $-80 \mathrm{mV}$, the sodium reversal potential is $50 \mathrm{mV}$, and the mixed cation reversal potential for $I_{\mathrm{H}}$ is $-20 \mathrm{mV}$. The leak reversal potential is $-50 \mathrm{mV}$.

Channel activation $(m)$ and inactivation $(h)$ are calculated as:

$$
\frac{d m}{d t}=\frac{m_{\infty}-m}{\tau_{\mathrm{m}}} ; \quad \frac{d h}{d t}=\frac{h_{\infty}-h}{\tau_{\mathrm{h}}} .
$$

All values for $m_{\infty}, h_{\infty}, \tau_{\mathrm{m}}, \tau_{\mathrm{h}}, p$, and the calcium dynamics are as described previously (Turrigiano et al., 1995; Liu et al., 1998) and reproduced here. The correctness of our implementation of the model was verified by comparing model output to published results (Table 1).

Calcium currents are calculated as:

$$
I_{\mathrm{Ca}}=\left(g_{\mathrm{CaT}}+g_{\mathrm{Cas}}\right)\left(V_{m}-E_{\mathrm{Ca}}\right) .
$$

Reversal potential for the calcium channels is calculated by the Nernst equation based on the current intracellular calcium concentration:

$$
E_{\mathrm{Ca}}=\left(\frac{\mathrm{RT}}{\mathrm{zF}}\right) \log \left(\frac{\mathrm{Ca}_{\text {out }}}{\mathrm{Ca}_{\text {in }}}\right),
$$

where $\mathrm{R}=8.314472(\mathrm{~J} / \mathrm{K} / \mathrm{mol}), \mathrm{T}=273.15+23.5^{\circ} \mathrm{K}, \mathrm{z}=+2, \mathrm{~F}=$ $9.649 \times 10^{4}(\mathrm{C} / \mathrm{mol})$. Calcium concentrations were calculated as in (Liu et al., 1998):

$$
\begin{aligned}
& \mathrm{d}\left(\mathrm{Ca}_{\text {in }}\right)=\mathrm{dt} \frac{(-0.94 \mu \mathrm{MnF} / \mathrm{nA}) \mathrm{I}_{\mathrm{Ca}}-\mathrm{Ca}_{\text {in }}+\mathrm{Ca}_{\text {internal_rest }}}{\mathrm{Ca}_{\text {buffering_rate }}}, \\
& \mathrm{Ca}_{\text {buffering_rate }}=20 \mathrm{~ms}, \mathrm{Ca}_{\text {out }}=3000 \mu \mathrm{M}, \mathrm{Ca}_{\text {internal_rest }}=0.05 \mu \mathrm{M} .
\end{aligned}
$$

To build a database of 1000 full-conductance models and 1000 reduced models, we began with two large sets of candidate models and then selected those that fired tonically at a rate of $3-7 \mathrm{~Hz}$, with a coefficient of variation $<0.05$ when injected with a current of $0.2 \mathrm{nA} / \mathrm{nF}$. The coefficient of variation was calculated from the last $2 \mathrm{~s}$ of simulation data from each model neuron. The candidate models were generated by randomly selecting active conductances from a uniform distribution ranging from 0.5 to $238 \mu \mathrm{S} / \mathrm{nF}$ for $\mathrm{Na}^{+}, K_{\mathrm{d}}$ $K_{\mathrm{Ca}}$, and $A$ and from 0.1 to $4.5 \mu \mathrm{S} / \mathrm{nF}$ for $\mathrm{Ca}_{\mathrm{T}}^{2+}, \mathrm{Ca}_{\mathrm{S}}^{2+}$, and $\mathrm{H}$ conductances. Leak was set to $0.01 \mu \mathrm{S} / \mathrm{nF}$ in all cases. Expression of conductances in units of $\mu \mathrm{S} / \mathrm{nF}$ (and similarly for currents as $\mathrm{nA} / \mathrm{nF}$ ) is used to normalize for cell membrane area. The full-conductance and reduced conductance candidate models were generated with statistically independent conductance values.

We simulated both sets of models at a range of current injection steps. The injected current, $I_{\text {inj }}$, ranged from $-2 \mathrm{nA} / \mathrm{nF}$ to $10 \mathrm{nA} / \mathrm{nF}$ with finer steps in the lower $I_{\text {inj }}$ values. Each model was run for $3 \mathrm{~s}$ of simulation time with a time step of $0.01 \mathrm{~ms}$. We then reran each model with the maximal sodium conductance tripled from its original value. For all models we recorded firing rate. For a subset of models we also recorded the voltage and channel state (activation and inactivation) history.

Analysis. All analysis was done with custom scripts written in MATLAB. Firing rate was calculated as the inverse of the mean interspike interval. The first second of data was discarded for each run to allow the simulation to equilibrate and frequency was only computed from the steady-state firing rate. Rheobase was computed as the lowest value of injected current that yielded a non-zero firing rate. Voltage threshold was computed at $10 \mathrm{nA} / \mathrm{nF}$ current injection as the point on the action potential rising phase where the derivative of the voltage trajectory reached $100 \mathrm{~V} / \mathrm{s}$. Voltage threshold was only computed in models that fired action potentials at $10 \mathrm{nA} / \mathrm{nF}$ current injection $(n=984)$. Gains were computed in two separate ways. When computed over the entire FI curve at one time we fit an exponential function times a line using the following equation:

$$
f(x)=\left(r_{\infty}+\left(r_{0}-r^{\infty}\right) e^{-\frac{x}{\tau_{r}}}\right)(m x+b) .
$$

To measure the gain we took the derivative of that function to yield:

$$
f^{\prime}(x)=m\left(\left(r_{0}-r_{\infty}\right) e^{-\frac{x}{\tau_{r}}}+r_{\infty}\right)+\frac{\left(r_{\infty}-r_{0}\right) e^{-\frac{x}{\tau_{r}}}(m x+b)}{\tau_{r}} .
$$




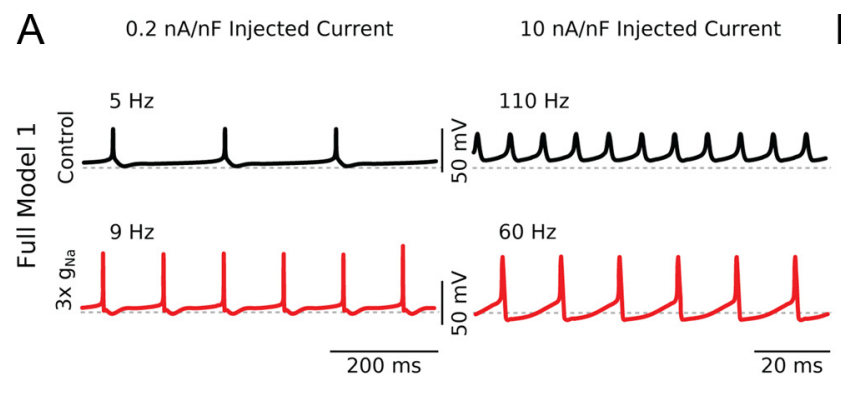

C $\quad 0.2 \mathrm{nA} / \mathrm{nF}$ Injected Current

$10 \mathrm{nA} / \mathrm{nF}$ Injected Current
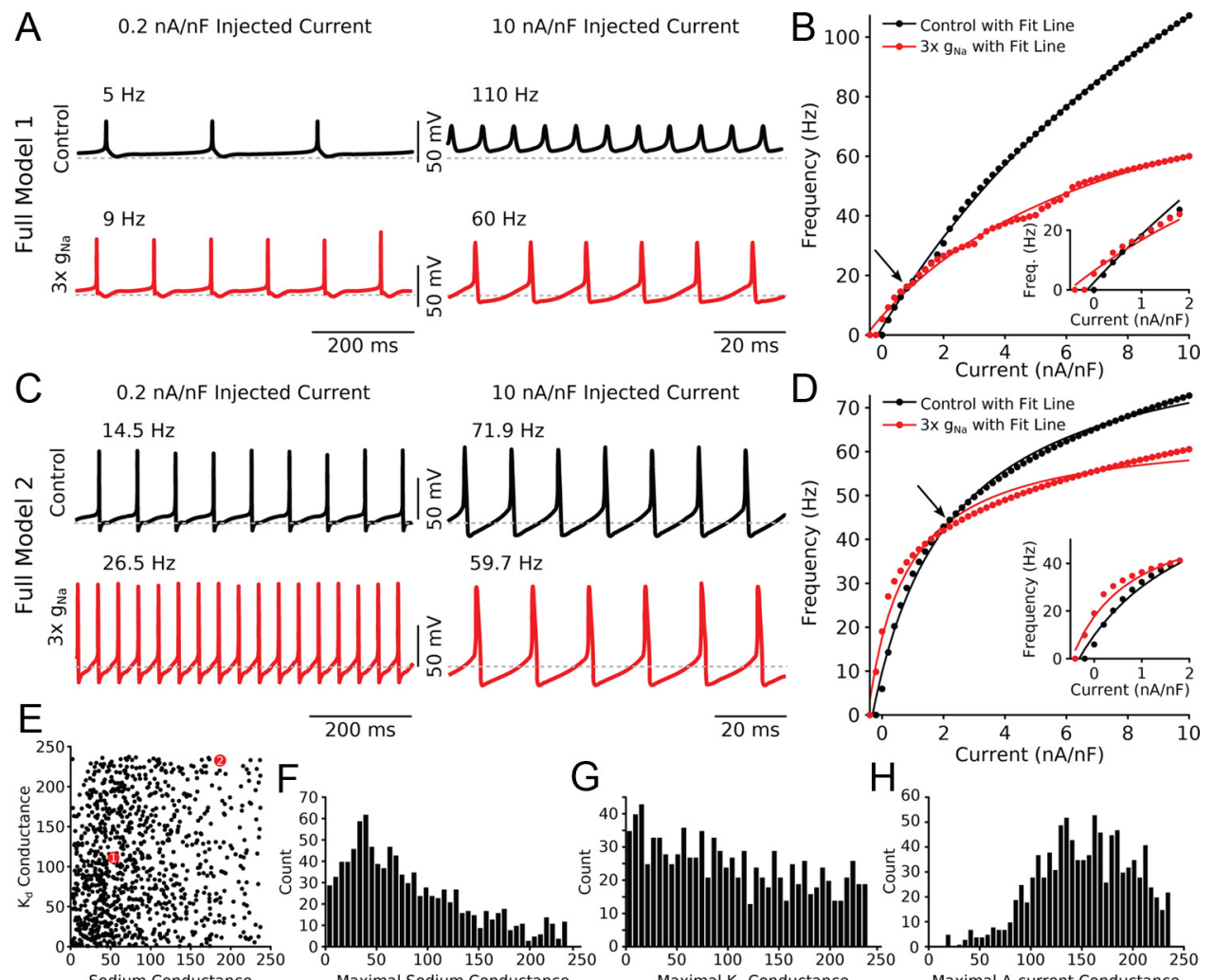

$\mathrm{H}_{60}$

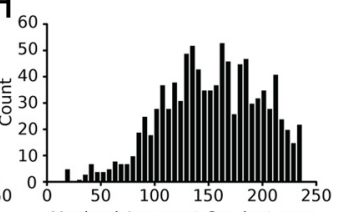

Figure 1. Full models show divisive gain change at high firing rates. $A$, Voltage traces from a single model neuron at low injected current $(0.2 \mathrm{nA} / \mathrm{nF}$, left column) and high injected current (10 $\mathrm{nA} / \mathrm{nF}$, right column) demonstrate that tripling the sodium conductance increases the firing rate at low current injection levels $(5 \mathrm{~Hz} \mathrm{to} 9 \mathrm{~Hz})$ but decreases the firing rate at higher current injection levels $(110 \mathrm{~Hz}$ to $60 \mathrm{~Hz})$. Red traces have tripled sodium conductance. $\boldsymbol{B}$, Fl curve for this example model shows enhanced excitability at low rates (inset) and divisive gain change at higher rates. Lines indicate fits to the data points (control, $\left.r^{2}=0.99 ; 3 \times g_{\mathrm{Na}} r^{2}=0.99\right)$. Arrow indicates crossover point. $\boldsymbol{C}, \boldsymbol{D}$, Same as $\boldsymbol{A}$ and $\boldsymbol{B}$ for another example model $\left(\right.$ control, $\left.r^{2}=0.99 ; 3 \times g_{\mathrm{Na}} r^{2}=0.98\right)$. $E$, Distribution of the sodium and $K_{\mathrm{d}}$ conductances for the entire 1000 model database. Each black dot represents one model. Models drawn as larger red circles represent the models shown in $A$ and $\boldsymbol{B}$ (model 1) and $\boldsymbol{C}$ and $\boldsymbol{D}$ (model 2). $\boldsymbol{F}$, Distribution of all the sodium conductances across the population. The selection criterion for this population favors lower values of maximal sodium conductance. $\mathbf{G}$, Distribution of all the maximal potassium $K_{\mathrm{d}}$ conductances across the population. $\boldsymbol{H}$, Distribution of all the maximal $A$-current conductances across the population.

In these equations $r_{\infty}$ is the asymptotic value of the exponential, $r_{0}$ is the initial value of the exponential and $\tau_{r}$ is the time constant of the exponential. At higher values of $x$ the curve is dominated by the line and the variable $m$ defines the slope and $b$ defines the $y$-intercept. In nearly all cases these functions fit FI curves extremely well with $r^{2}$ values $>0.95$. When fitting only the high and low current injection regions of the FI curve, we fit line segments because fitting a line to a restricted section of the FI curve resulted in fits with lower errors. One-tailed $t$ tests were used when sample means were compared to zero and a paired $t$ test was used when comparing paired samples. Histograms were fit with normal distributions for comparison regardless of whether those distributions were Gaussian. Figures were generated in MATLAB and finished in Inkscape (inkscape.org).

\section{Results}

\section{Firing rate change in a biophysically complex model}

We constructed a population of 1000 model neurons, each with 7 separate active conductances similar to those used by Liu et al. (1998), and based on voltage-clamp data reported by Turrigiano et al. (1995) (see Materials and Methods). In this population the maximal conductances were randomly assigned. For each model generated in this fashion, we determined whether it would generate tonic spiking when a current of $0.2 \mathrm{nA} / \mathrm{nF}$ was injected. Only models that showed stable tonic firing (coefficient of variation of its spike train $<0.05$ ) were included in this population.

We initially subjected model neurons to a range of biophysical changes that have been observed experimentally in response to longterm sodium blockade (Desai et al., 1999). This included reducing the delayed rectifier potassium conductance $\left(K_{\mathrm{d}}\right)$, increasing the sodium conductance, or both. We noticed unexpected firing rate decreases for large increases in the sodium conductance at high current injection levels and thus chose to study this manipulation in detail. While our choice to triple the sodium conductance parameter was arbitrary, using a large change yields a consistent effect that is easier to study than more subtle manipulations in a highly variable population. Smaller changes similar to those observed experimentally (Desai et al., 1999) yielded a proportional effect size suggesting that our results are not dependent on a large manipulation (data not shown).

To assess model excitability over a range of input currents we measured the FI curve for all models in the population. In one example full conductance model neuron (Fig. $1 A$ ), the firing rate increased with low current injection $(0.2 \mathrm{nA} / \mathrm{nF})$ when sodium conductance was increased by a factor of 3 (Fig. $1 A$, left traces, black: control, red: $\left.3 \times g_{\mathrm{Na}}\right)$. For high current injection into the same model neuron (10 nA/nF), the opposite occurred (Fig. $1 A$, right traces) and increasing the $\mathrm{Na}^{+}$conductance led to decreased firing rates. The FI curves for this neuron (Fig. $1 B$ ) intersect near the middle of the dynamic range (arrow). A second example full conductance neuron (Fig. 1C) from the population has different firing behavior because it has different maximal conductances but retains the same qualitative changes to its excitability (Fig. 1D) in response to $\mathrm{Na}^{+}$conductance increases and thus also has FI curves that intersect (arrow). 

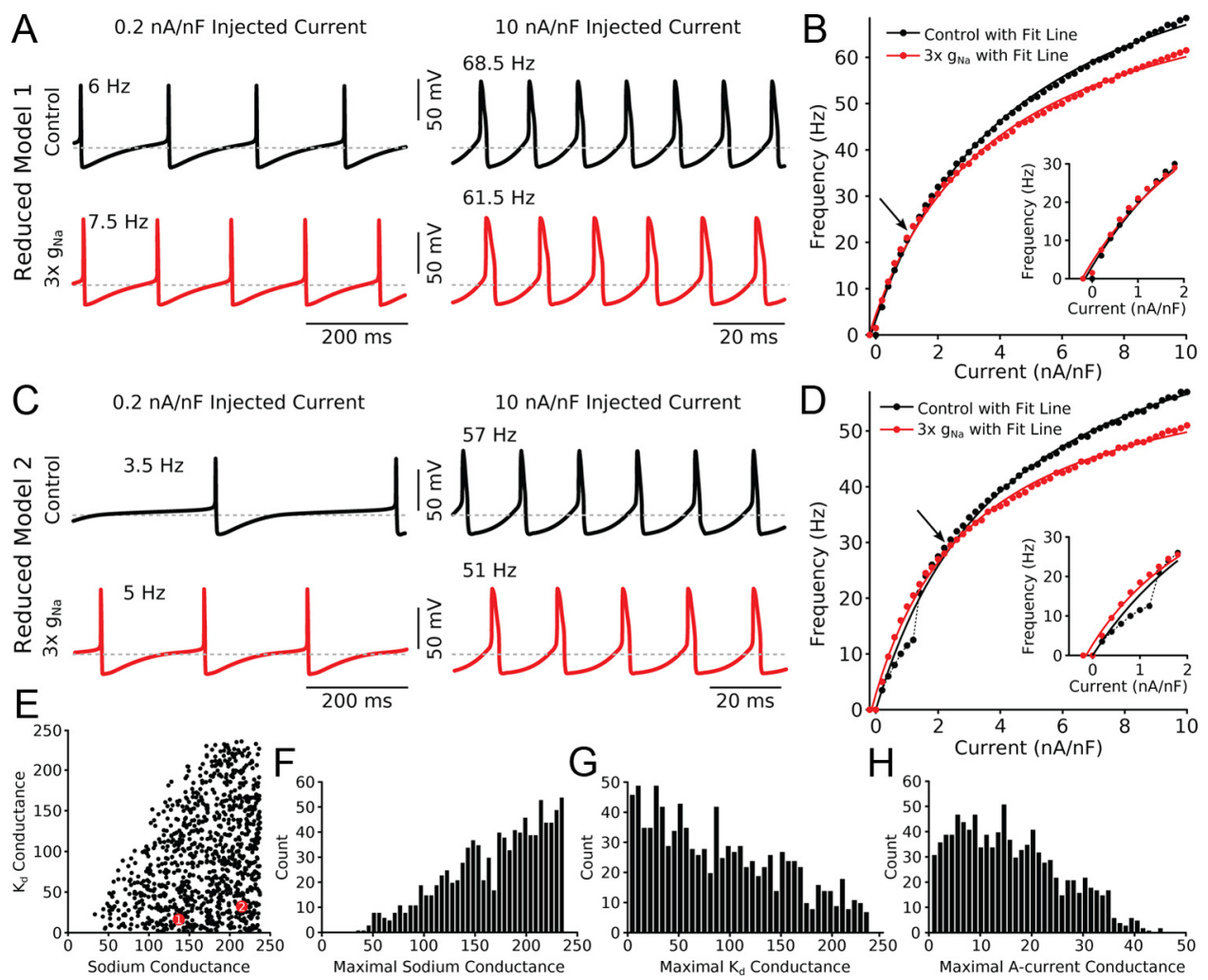

Figure 2. Reduced models show divisive gain change at high firing rates. $A$, Voltage traces from a single reduced model neuron at low injected current $(0.2 \mathrm{nA} / \mathrm{nF}$, left column) and high injected current $(10 \mathrm{nA} / \mathrm{nF}$, right column) demonstrate that tripling the sodium conductance increases the firing rate at low current injection levels $(6-7.5 \mathrm{~Hz})$ but decreases the firing rate at higher current injection levels ( $68.5-61.5 \mathrm{~Hz}$ ). Red traces have tripled sodium conductance. $\boldsymbol{B}$, Fl curve for this example model shows enhanced excitability at low rates (inset) and divisive gain change at higher rates. Lines indicate fits to the data points (control, $\left.r^{2}=0.99 ; 3 \times g_{\mathrm{Na}}, r^{2}=0.99\right)$. Arrow indicates crossover point. $\boldsymbol{C}, \boldsymbol{D}$, Same as $\boldsymbol{A}$ and $\boldsymbol{B}$ for an additional example model $\left(\right.$ control, $r^{2}=0.98 ; 3 \times$ $g_{\mathrm{Na}} r^{2}=0.99$ ). Black dotted line in $\boldsymbol{D}$ (main panel and inset) connects individual control data points and highlights that there are models that experience rapid changes in frequency over the input current range. $E$, Distribution of the sodium and $K_{\mathrm{d}}$ conductances for the entire, 1000 model database. Each black dot represents one model. Models drawn as larger red circles represent the models shown in $\boldsymbol{A}$ and $\boldsymbol{B}$ (model 1 ) and $\boldsymbol{C}$ and $\boldsymbol{D}$ (model 2). $\boldsymbol{F}$, Distribution of all the sodium conductances across the population. The selection criterion for this population favors higher values of maximal sodium conductance. $\boldsymbol{G}$, Distribution of all the maximal $K_{\mathrm{d}}$ conductances across the population. The selection criterion for this population favors lower values of maximal $K_{\mathrm{d}}$ conductance. $\boldsymbol{H}$, Distribution of all the maximal $A$-current conductances across the population.

As expected, in this database of biophysically complex models with randomly generated conductances (Fig. $1 E-H$ shows the range of parameters present in the population), we observed a wide variety of spiking behaviors and FI curves (Liu et al., 1998; Goldman et al., 2001). For many models in the database ( $~ 300$ of 1000 models), high input currents lead to a reduction in the gain, a multiplicative scaling operation thought to underlie important neuronal computations (Salinas and Thier, 2000; Gabbiani et al., 2002; Silver, 2010). At low firing rates, increased $\mathrm{Na}^{+}$conductance usually ( $\sim 970$ of 1000 models) led to a decrease in the rheobase of the neuron. Qualitatively, this meant that the firing rate of the models with high $\mathrm{Na}^{+}$conductance was increased at smaller input current, but decreased at larger input current relative to control which lead to the FI curves intersecting (Fig. $1 B, D$, arrows). The change in rheobase could be explained easily since enhanced $\mathrm{Na}^{+}$conductance leads to a hyperpolarized voltage threshold allowing a smaller amount of input current to drive spiking in the model. The decreased gain at higher input current, however, required a more detailed analysis of the interaction between the sodium and delayed rectifier potassium conductances which we address later.

Reduced model database shows subtractive shift at low rates and divisive change at high rates

Next, we sought to understand whether the above results could be reproduced in a more simplified biophysical model. Reduced models are more amenable to detailed biophysical analysis and allow for the precise identification of mechanisms that underlie specific behaviors. The reduced model we generated contained only $\mathrm{Na}^{+}\left(I_{\mathrm{Na}}\right)$ and delayed rectifier potassium $\left(I_{\mathrm{Kd}}\right)$ conductances along with an $A$-type potassium $\left(I_{\mathrm{A}}\right)$ and leak conductance (see Materials and Methods). While the $I_{\mathrm{Na}}$ and $I_{\mathrm{Kd}}$ are responsible for the spike generating mechanism, $I_{\mathrm{A}}$ promotes type $1 \mathrm{FI}$ curve dynamics, such that the model neuron can fire at arbitrarily low rates (Connor and Stevens, 1971; Connor et al., 1977; Dayan and Abbott, 2001). We selected this model because it is a generic representation of the core features of many types of spiking neurons. Thus, these results should apply broadly to neurons with a spiking mechanism similar to the one in this model. All further results presented in this study were generated with the reduced model.

We next generated a population of 1000 reduced models each with random conductances and selected to have tonic firing at 0.2 $\mathrm{nA} / \mathrm{nF}$ current injection. In an example reduced neuron (Fig. $2 \mathrm{~A}$ ) we observed the same firing rate changes reported for the fullconductance models and the same qualitative features in the FI curves (Fig. $2 B$ ). An additional example model neuron also had similar firing (Fig. 2C) and FI curve properties (Fig. 2D). We measured the FI curves of all the models in the population (Fig. $2 E-H$, range of parameters present in the population) and repeated this process after increasing the maximal $\mathrm{Na}^{+}$conductance by a factor of three. As in the full conductance models, this 
A

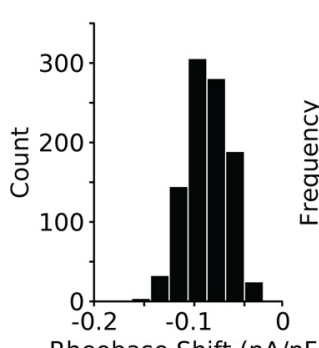

Rheobase Shift $(\mathrm{nA} / \mathrm{nF})$
B

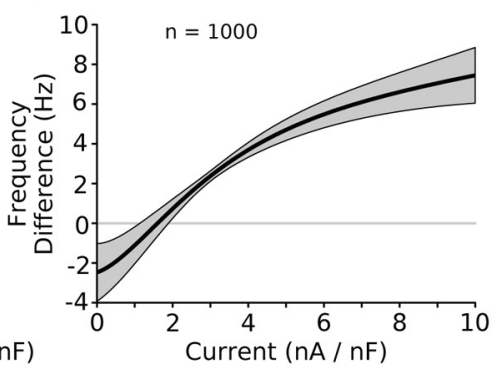

C

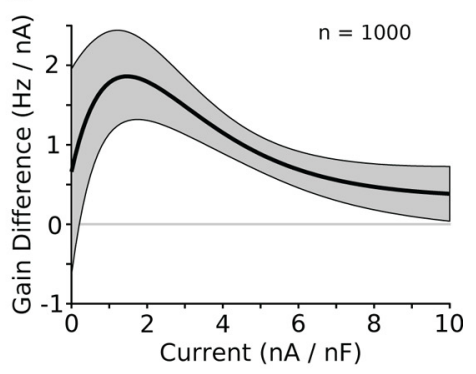

High Rate

F

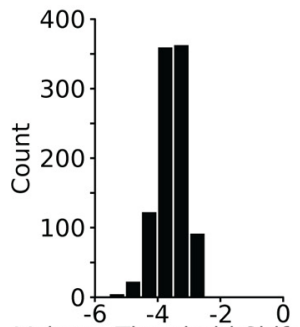

Voltage Threshold Shift $(\mathrm{mV})$

\section{E}
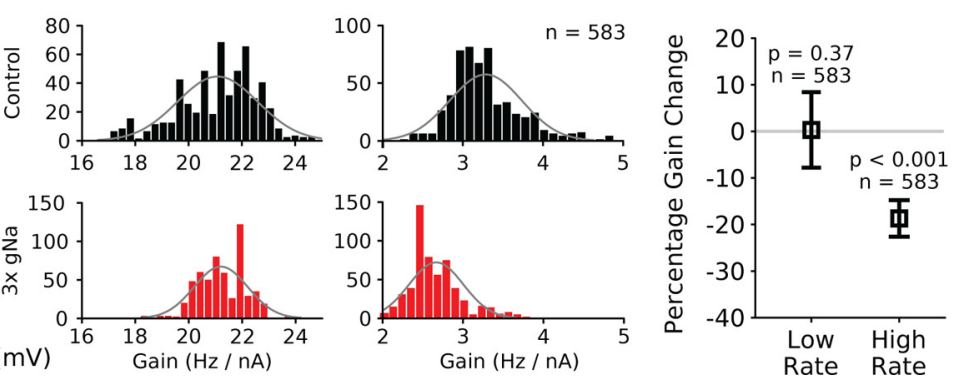

Figure 3. Enhanced sodium conductance causes a subtractive shift at low rates and a divisive gain change at high rates. $\boldsymbol{A}$, The rheobase (current threshold) decreases in all models when sodium conductance is increased. Histogram plots values by which rheobase shifts when going from control to $3 \times g_{\mathrm{Na}}$. All shifts are negative indicating that the shift occurs in all 1000 models. $\boldsymbol{B}$, Subtraction of Fl curve fits (control $-3 \times g_{\mathrm{Na}}$ ) show that the difference is reliably negative at low rates and positive at high rates suggesting a subtractive shift at low rates, a crossover point and a divisive gain change at high rates. Gray area represents 1 SD $(n=1000)$. C, Subtraction of the derivatives of the Fl curve fits shows the input current at which the models experience the largest gain difference. Gray area represents 1 SD $(n=1000)$. $\boldsymbol{D}$, Voltage threshold is hyperpolarized in all models that spike at high current injection $(n=984)$ when Na ${ }^{+}$conductance is increased. $\boldsymbol{E}$, Histograms of gain distributions of low and high firing rates for each model under control and $3 \times g_{\mathrm{Na}}$ conditions. At low firing rates, the distributions have similar means while at high rates, gain is reduced (black, control; red, $3 \times g_{\mathrm{Na}^{\prime}} n=583$ ). Model population was subselected to ignore Fl curves with jumps in frequency that correspond to missed spikes. Gray lines are Gaussian distributions with the same means and variances as the data in the histograms. $\boldsymbol{F}$, Gain changes significantly at high rates but not at low rates. Gain changes are plotted as percentages to normalize between low and high firing rates (low rate, $0.3 \pm 8.1 \%, p=0.37, n=583$; high rate, $-18.7 \pm 3.9 \%, p<0.001, n=583$, one-sample $t$ test).

population showed an increase in the excitability of the neuron at low firing rates when $\mathrm{Na}^{+}$conductance was tripled. As the input current increased, additional $\mathrm{Na}^{+}$conductance decreased the firing rate for equivalent input current (Fig. $2 B, D$, red FI curves). As before, this meant that the two FI curves intersected one another (Fig. $2 B, D$, arrows).

These observations indicated that the fundamental mechanisms that were responsible for generating a subtractive shift in rheobase and a divisive change in gain at high firing rates under conditions of high $\mathrm{Na}^{+}$conductance were present in this reduced model. Furthermore, compared with the full model, the divisive gain change in the reduced model was highly robust and present in the majority of the models generated in this manner (984 of 1000 models) even though the conductances of individual models were highly variable (Fig. $2 E-H$ ).

Finally, to ensure that our results were not dependent on very large changes to the sodium conductance, we reran our simulations with conductance changes more similar to those observed experimentally. After activity deprivation, cultured neurons have been reported to undergo a $1.3 \times$ increase in their sodium conductance (Desai et al., 1999). When increasing the sodium conductance by this amount we observed approximately a $25 \%$ decrease in the gain compared with the full effect (data not shown) suggesting that the reported effect size is proportional to the sodium conductance change and that our results were not dependent on a large manipulation size.

To quantify these observations across the population, we first determined whether increasing the $\mathrm{Na}^{+}$conductance by a factor of three in the population of reduced models changed the rheobase. When $\mathrm{Na}^{+}$conductance was increased, rheobase was de- creased in all models in the population (Fig. $3 A$, rheobase shift from control to $3 \times g_{\mathrm{Na}}$ averaged $\sim-0.1 \mathrm{nA} / \mathrm{nF}$ ). To measure the change in excitability generated over the entire input current range we fit an exponential function multiplied by a line to the FI curves from the population (see Materials and Methods). In general, this function fit the FI curves extremely well $\left(r^{2}>0.95\right.$ in most models). For each model we fit both the control and $3 \times$ $\mathrm{Na}^{+}$conductance FI curves and then subtracted the two fit lines from one another (control minus enhanced $\mathrm{Na}^{+}$conductance). When these differenced fit lines were averaged over the entire population, a picture of the changes in the excitability emerged (Fig. 3B, black line, mean; gray region, $\pm 1 \mathrm{SD}, n=1000$ ). As expected, at low current injection levels the mean difference was negative indicating that the firing rate was higher with enhanced sodium conductance than in the control condition. Conversely, at higher current injection levels the value of the difference curve was positive, indicating that the control FI curve had higher firing rates in this range. Finally, the current injection level that yielded a zero frequency difference value corresponds to the crossover point of the FI curves and fell near $1.5 \mathrm{nA} / \mathrm{nF}$ current injection.

Fitting the FI curves also allowed computing the gain by taking the derivative (the slope) of the fit line equation (see Materials and Methods). To measure where the gains of the FI curves were most different, we subtracted the derivatives of the control and $3 \times g_{\mathrm{Na}}$ FI curves from one another (Fig. $3 C$, black line, mean; gray region, $\pm 1 \mathrm{SD}, n=1000$, control minus enhanced sodium conductance). The maximum gain difference was near $1.5 \mathrm{nA} / \mathrm{nF}$ current injection, very close to the crossing over point (Fig. $3 B$, zero crossing). We also computed the change in voltage threshold in the population by measuring when the rate of rise of an action 


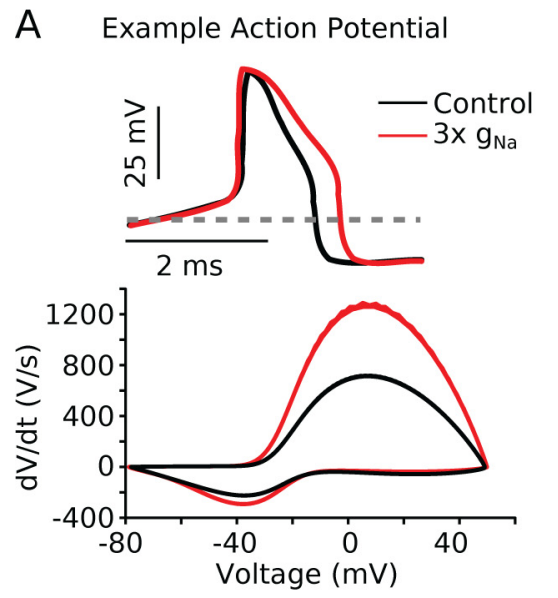

E

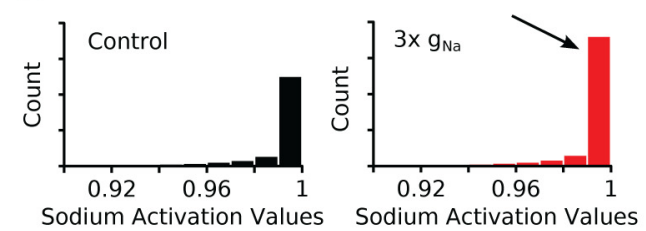

B

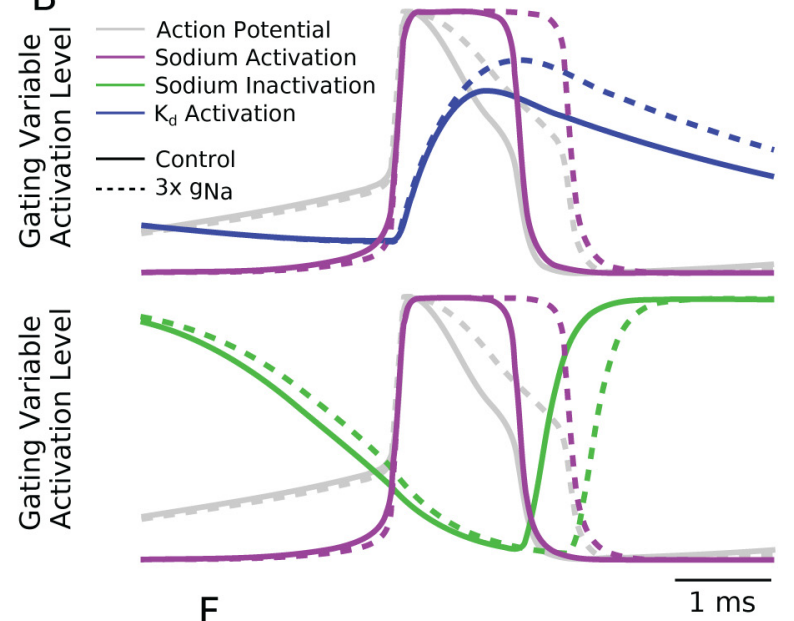

F

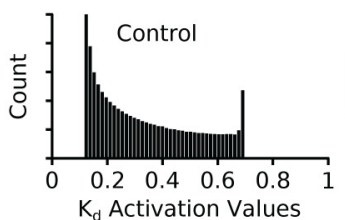

C
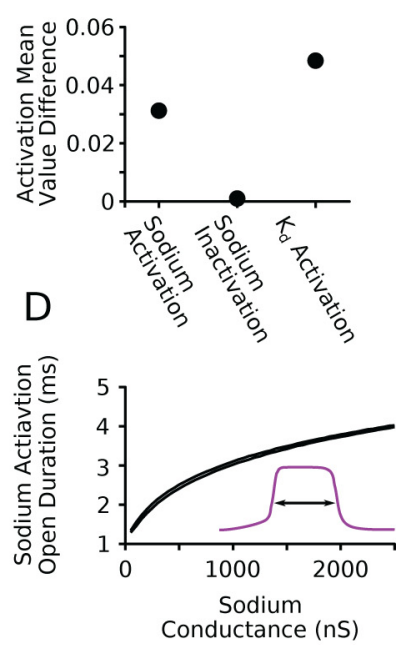

Figure 4. Sodium conductance changes lead to a proportionally greater activation of $K_{\mathrm{d}}$ conductance which lowers the firing rate overall. $\boldsymbol{A}$, Top, An example action potential from a single model being injected with $10 \mathrm{nA} / \mathrm{nF}$ of current from the population (model 1 from Fig. 2) was overlaid from both control and $3 \times g_{\mathrm{Na}}$ conditions. Enhanced sodium conductance widens the action potential. Bottom, A phase plot of the same action potential shows that the voltage initially rises faster and then repolarizes more slowly followed by a final phase of more rapid repolarization when compared with control. $\boldsymbol{B}$, Gating variables during the action potential shown in $\boldsymbol{A}$. The normalized action potential is plotted in gray for comparison. Solid lines represent control; dotted lines represent $3 \times$ $g_{\mathrm{Na}}$. Top, High sodium activation is prolonged (purple dotted line). This leads to enhanced activation of the $K_{\mathrm{d}}$ conductance (blue dotted line). Bottom, No major changes to the amplitude of the sodium inactivation are observed (green). $C$, The difference of gating variable values ( $3 \times g_{\mathrm{Na}}$ minus control) shows that while the wider spikes lead to greater mean $g_{\mathrm{Na}}$ activation, the $K_{\mathrm{d}}$ activation variable is enhanced proportionally more. There is no change in the mean level of activation for the sodium inactivation. Values are adjusted for firing rate. $D$, The time spent by the sodium activation in the open state is prolonged continuously when the sodium maximal conductance is increased up to 10 -fold ( $2500 \mathrm{nS}$ ). Two (nearly identical) lines are plotted corresponding to the two models shown in Figure 2. Inset, Schematic of the quantity being measured, the time when the sodium activation variable value is $>0.96$ during an action potential. $\boldsymbol{E}$, Histogram of sodium channel activation variable values over a train of action potentials (black, control; red, $3 \times g_{\mathrm{Na}}$ ). Arrow highlights that with $3 \times g_{\mathrm{Na}}$ the gate is more likely to be open (a taller bar indicates more time spent in the open state). Axis is zoomed in to highlight high values of activation. $\boldsymbol{F}$, Histogram of $K_{\mathrm{d}}$ activation variable values during a train of action potentials (black, control; red, $3 \times g_{\mathrm{Na}}$ ). Arrow highlights that with $3 \times g_{\mathrm{Na}}$ the $K_{\mathrm{d}}$ activation has consistently higher values indicating increased open probability.

potential exceeded $100 \mathrm{~V} / \mathrm{s}$. In all models that spiked at high current injection, we observed a decrease in the voltage threshold, indicating that spikes could be initiated from a lower starting voltage when $\mathrm{Na}^{+}$conductance was tripled (Fig. 3D, voltage threshold shifted $\sim-4 \mathrm{mV}$ from control to $\left.3 \times g_{\mathrm{Na}}, n=984\right)$.

The mean gain difference at low current injection was $\sim 1$ $\mathrm{SD}$ above zero (Fig. $3 C$, gray region), which seemed large given the similarity of many FI curves in this range (Fig. $2 B$ ). The large difference could be explained by observing that several control FI curves had low gain at low input current but large and abrupt changes in gain as input current was increased (see Fig. $2 D$, dotted line, for an example; effect occurs in 417 models out of 1000). Inspection of voltage traces determined that this phenomenon was because these models had failed spikes at low current injection levels during which the neuron would depolarize to near threshold, nearly spike, and then return to rest. As input current increased, these perithreshold depolarizations turned into action potentials, rapidly increasing the firing rate.

We subselected those models from the population that displayed tonic spiking at all low current injection levels (not just at the initial level of $0.2 \mathrm{nA} / \mathrm{nF}$ ). In those 583 models we fit line segments to both the control and $3 \times \mathrm{Na}^{+}$conductance FI curves at both low and high current injection and compared the slopes (Fig. 3E). For this analysis, we fit line segments because the low and high regions of the FI curves were not well fit by the curves used previously. The slopes of the line segments at low rates were very similar (Fig. 3E, left column, control, $21.9 \pm 1.5 \mathrm{~Hz} / \mathrm{nA} ; 3 \times$ $\left.\mathrm{Na}^{+}, 21.2 \pm 1.0 \mathrm{~Hz} / \mathrm{nA}, n=583\right)$, whereas a noticeable difference was present in the slopes at higher rates (Fig. $3 E$, right column, control, $\left.3.3 \pm 0.5 \mathrm{~Hz} / \mathrm{nA} ; 3 \times \mathrm{Na}^{+}, 2.7 \pm 0.3 \mathrm{~Hz} / \mathrm{nA}, n=583\right)$. We measured the percentage change in each model at low and high rates and found that there was no significant change at low rates (Fig. $3 F$, low rate, $0.3 \pm 8.1 \%, p=0.37, n=583, t$ test) whereas the slope was significantly changed at high firing rates (Fig. $3 F$, high rate, $18.7 \pm 3.9 \%, p<0.001, n=583$, $t$ test). Together, these results show that the population of models that fired tonically through the entire low-frequency range had a subtractive shift in their FI curves with no slope change at low rates and a divisive change in gain at high firing rates all in response to a single conductance change.

\section{Mechanism of gain change at higher firing rates}

To understand how the targeted change to the $\mathrm{Na}^{+}$conductance was producing a divisive change in gain at higher firing rates in the reduced model neurons, we investigated the activation level of the individual spiking conductances during an action potential with $10 \mathrm{nA} / \mathrm{nF}$ current injection. We first compared a spike from a representative model neuron (Fig. 2, model 1 shown) before and after changes to the $\mathrm{Na}^{+}$conductance (Fig. $4 A$, top). There was no change in mean voltage with increased $\mathrm{Na}^{+}$conductance computed over a single interspike interval (data not shown). However, we did notice that individual spikes became wider with increased $\mathrm{Na}^{+}$conductance. Additionally, increasing the $\mathrm{Na}^{+}$ 
conductance hyperpolarized the voltage threshold for spike initiation, as we previously noted (Fig. $3 D$ ).

To more quantitatively analyze the change in spike width we plotted the change in voltage relative to the voltage (Fig. $4 \mathrm{~A}$, bottom). In both cases, the most depolarized voltage value achieved remained approximately unchanged (near the sodium reversal potential). With enhanced $\mathrm{Na}^{+}$conductance, single action potentials depolarize more rapidly (a higher rate of rise). Conversely, the repolarization of the spike on the downstroke phase was initially slower than control, but the final phase of repolarization was more rapid than control leading to an overall lengthening of the spike.

To understand how increased sodium conductance and wider spikes were leading to a reduction in firing rate (equivalent to an increase in the interspike interval) at high current injection, we plotted the sodium channel activation variable [ $m$ in the classic Hodgkin and Huxley formalism (Hodgkin and Huxley, 1952)], the sodium channel inactivation variable $(h)$ and the $K_{\mathrm{d}}$ channel activation variable ( $n$ ) during a single action potential (Fig. $4 B$ ). We compared traces from control conditions (Fig. 4B, solid lines) with those generated after the $\mathrm{Na}^{+}$conductance had been increased threefold (Fig. 4B, dashed lines). We lined up the traces based on the rising phase of the $\mathrm{Na}^{+}$channel activation variable (Fig. $4 \mathrm{~B}$, purple lines) and superimposed the corresponding action potential with voltage normalized to be between 0 and 1 (Fig. $4 B$, gray traces, same spike as in Fig. $4 A$ ). Because the increased sodium conductance prolongs the depolarization of the neuron, keeping the sodium channels open longer (Fig. $4 B$, purple, top and bottom), more time must elapse before sufficient sodium channels are inactivated to reduce the inward current to a point where the $K_{\mathrm{d}}$ current can effectively oppose it. This change by itself was sufficient to explain the wider spikes but not the increased interspike interval.

The change in the interspike interval can be explained by comparing the action potential voltage trajectory with the $K_{\mathrm{d}}$ channel activation (Fig. $4 B$, blue, top). The $K_{\mathrm{d}}$ channel has slow activation kinetics when compared with the sodium channel. Because of the prolonged depolarization of the neuron during an action potential when $\mathrm{Na}^{+}$conductance was tripled, the $K_{\mathrm{d}}$ channels had more time to activate, reaching a higher maximum activation level during a spike (Fig. $4 B$, blue, top). The slow decay of the $K_{\mathrm{d}}$ activation variable is ultimately responsible for increasing the interspike interval (lowering the firing rate). Contrary to $K_{\mathrm{d}}$, the $\mathrm{Na}^{+}$inactivation (Fig. $4 \mathrm{~B}$, green, bottom) had little change in its amplitude or kinetics and simply shifted its trajectory to match the wider action potential.

We quantified the change in open probability normalized for firing rate of these three gates between control and enhanced $\mathrm{Na}^{+}$conductance. We found that for any changes in the $\mathrm{Na}^{+}$ activation there was a disproportionate increase in the $K_{\mathrm{d}}$ open probability (Fig. 4C). In contrast, there was no change in the open probability of the $\mathrm{Na}^{+}$inactivation gate between the two conditions tested. Thus the lower firing rate observed with enhanced $\mathrm{Na}^{+}$maximal conductance is due to a prolonged depolarization of the voltage that leads to a greater activation of $K_{\mathrm{d}}$ ultimately slowing the overall firing rate.

Because greater spike width due to larger $\mathrm{Na}^{+}$conductance seemed to be critical for firing rate changes via enhanced $K_{\mathrm{d}}$ channel activation, we ran two example models (the models pictured in Fig. 2) over a much larger maximal sodium conductance parameter range (Fig. $4 D$, figures shows two nearly superimposed curves). The model population had sodium conductances ranging between 0.5 and $250 \mathrm{nS} / \mathrm{nF}$ under control. We further extended this range to cover a tenfold increase ranging from 5 to $2500 \mathrm{nS} / \mathrm{nF}$ and measured the length of time the $\mathrm{Na}^{+}$activation gate stayed open during an action potential (Fig. $4 D$, inset schematic, double arrow indicates measured quantity). As expected, this duration continued to increase with continually increased maximal $\mathrm{Na}^{+}$conductance, providing evidence that the explanation for how more $\mathrm{Na}^{+}$conductance can lead to lower firing rates is accurate in this model.

Finally, we plotted histograms of the values of each activation variable over a train of action potentials to visualize more clearly how the $\mathrm{Na}^{+}$conductance change influenced the open probabilities of each gate. These histograms count the frequency of each value of the activation variables and thus indirectly measure how long the gate spends in the open state. The $\mathrm{Na}^{+}$activation rapidly switched between the entirely open or closed configuration. When the $\mathrm{Na}^{+}$conductance was increased, the activation variable spent more time in the open state (Fig. $4 E$, arrow), which can be seen as an increase in the height of the bar at values near 1.0. The values of the $K_{\mathrm{d}}$ activation were greater overall with increased $\mathrm{Na}^{+}$conductance than those observed under control (Fig. $4 F$, arrow), indicating that the $K_{\mathrm{d}}$ conductance was activated more strongly. Together, these results show that increasing the $\mathrm{Na}^{+}$ conductance enhances the proportion of a spike phase that the $\mathrm{Na}^{+}$channel activation spends in the fully open state (Fig. $4 E$, arrow). Enhanced activation leads to a larger activation of the $K_{\mathrm{d}}$ conductance (Fig. $4 F$, arrow), ultimately leading to a reduction of the firing rate by delaying the subsequent spikes.

\section{Crossover point variance suggests a more complex homeostatic regulator}

As mentioned above, a consequence of the observed ratedependent FI curve modulation is that the control and enhanced $\mathrm{Na}^{+}$FI curves intersect. The intersection point represents the input current and frequency value at which the subtractive shift in the FI curve transitions into a divisive gain change. We measured how the crossover point changes between each model in the database of 1000 reduced model neurons. For each set of FI curves (control and threefold increased $\mathrm{Na}^{+}$conductance) we used the fit lines computed previously and calculated where these two curves intersected. We found variance in the distribution of the crossover points, both in terms of the current values and the frequency values at which they occurred (Fig. 5). This indicated that there was no fixed current or frequency value that delimits the regions in which enhancing the $\mathrm{Na}^{+}$conductance either produces a subtractive shift in the FI curve or a divisive gain change. We binned both the current and frequency values of the transition and found that the current values had a large peak at $\sim 1.4 \mathrm{nA}$ with a tail at higher current values and that the frequency values were roughly normally distributed (Fig. 5, histograms, gray lines are normal distributions with mean and variance equal to the data in the histograms). In general, these findings indicate that a homeostatic regulator of excitability cannot be implemented based on fixed readouts because these values will vary in real neurons.

\section{Discussion}

Nervous systems show remarkable resilience in their ability to remain functional when perturbed by neuromodulators (Marder and Bucher, 2007) and by temperature changes (Rosbash, 2009; Tang et al., 2010, 2012). Responses to such global changes and more targeted genetic or pharmacological manipulations are often modest, suggesting that compensatory or degenerate mechanisms are a core feature of nervous systems. Neurons in 


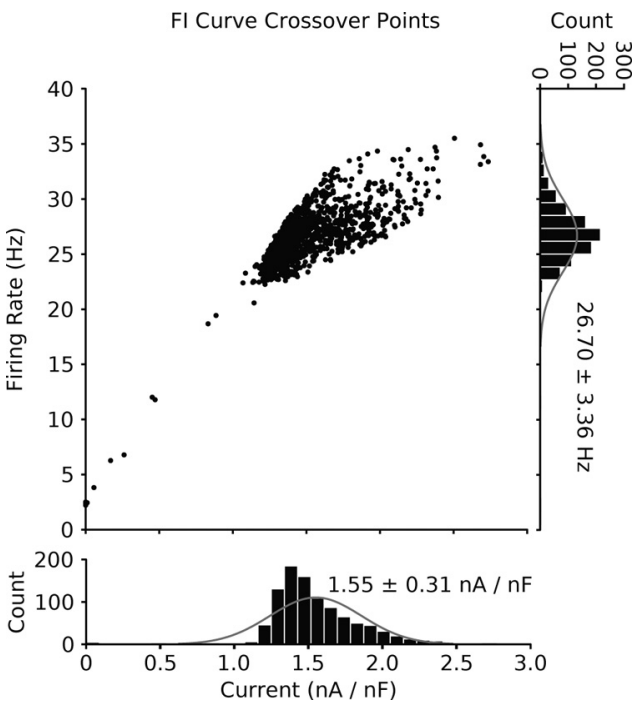

Figure 5. Fl curve crossover point occurs at diverse values of both frequency and current. The crossover points for all the reduced models are plotted in input current vs frequency space $(n=$ $\sim 1000$, few models had no intersection or extreme values for their crossover points and are omitted for clarity). The crossover point represents the value of current and frequency that separates the two effects enhanced sodium conductance has on the control FI curve. The scatter plot highlights that the crossover points from each model occur at many different values of frequency and current. Histograms of the current values (bottom, $1.55 \pm 0.31 \mathrm{nA} / \mathrm{nF}$ ) and frequency values (right, $26.7 \pm 3.36 \mathrm{~Hz}$ ) are plotted along with representative normal distributions (gray lines) that have equivalent means and variances.

functioning circuits are faced with the task of continuously modulating their excitability in response to changing inputs and altered environmental conditions. In this study, we asked how targeted increases in the number of sodium channels would influence the excitability of a model neuron. At higher input currents, increased $\mathrm{Na}^{+}$conductance led to a surprising reduction of the firing rate and the outcome of $\mathrm{Na}^{+}$conductance increase depended on the firing rate of the neuron. We conclude that this phenomenon poses a difficult problem for neurons attempting to maintain their excitability by changing conductance densities and suggests they must not only match a target level of mean activity but also match a target input-output relationship.

\section{Sodium conductance changes as a mechanism for gain control}

The experimental and theoretical study of gain control has elucidated in detail how the properties of synaptic input trains alter neuronal gain (Destexhe et al., 2003) and how some biophysical properties influence the shape of the input-output relationship (Desai et al., 1999; Lundstrom et al., 2008, 2009; O'Leary et al., 2010). Neurons in vivo are subject to high rates of synaptic input that introduce membrane voltage fluctuations and membrane conductance changes (Paré et al., 1998; Destexhe and Paré, 1999; Harvey et al., 2009). These two properties of in vivo neurons have been shown to have a significant influence on the FI curve (Holt and Koch, 1997; Chance et al., 2002; Mitchell and Silver, 2003; Prescott and De Koninck, 2003; Prescott et al., 2006). Alternate mechanisms for gain control such as synapses with short-term depression have also been proposed as mechanisms for multiplicative gain control (Abbott et al., 1997; Rothman et al., 2009). Comparatively little attention, however, has been focused on cell-autonomous methods of gain control such as the regulation of intrin- sic conductances (Moyer et al., 1996; Cantrell and Catterall, 2001; Zhang and Linden, 2003; Schulz, 2006).

We present a population of model neurons in which the gain is divisively scaled at higher firing rates while there is a subtractive shift in the FI curve at low rates, both in response to an increase in the $\mathrm{Na}^{+}$conductance. This mechanism is significant because it represents an intrinsic form of gain control in which the neuron can itself influence its input-output relationship by changing its intrinsic properties rather than integrating synaptic inputs over which it has no direct control.

The idea that the entire input-output property of a neuron is the key quantity that must be homeostatically regulated has been proposed previously (Stemmler and Koch, 1999; Triesch, 2007; Lundstrom et al., 2008, 2009). These previous studies have related basic biophysical properties such as conductance ratios (Lundstrom et al., 2008), timescales of biophysical variables (Lundstrom et al., 2009), maximization of information transfer (Stemmler and Koch, 1999) and the statistical distribution of inputs (Triesch, 2007; Wark et al., 2007) to the FI curve. This study has implications for defining the properties of a homeostatic mechanism that could regulate the entire input-output property of neurons, not just the mean firing rate. Additionally, our study reinforces the idea that neurons in functioning networks cannot always rely on single conductance changes to have a single effect on excitability.

The dynamics of sodium channel activation and inactivation are complex (Carter and Bean, 2011), as are the effects of sodium conductance changes on excitability. Upregulation of the sodium conductance can lead to increases in excitability (Hodgkin, 1975; Cantrell and Catterall, 2001) but also decreases (Günay et al., 2008). Somatic excitability is enhanced by dendritic sodium channel block in pyramidal neurons of the weakly electric fish (Fernandez et al., 2005), chronic tetrodotoxin (TTX) treatment leads to decreased EPSC frequency at hippocampal CA3 recurrent synapses (Kim and Tsien, 2008), blocking activity in layer 5 cortical interneurons with TTX enhances pyramidal cell dendritic responses (Murayama et al., 2009), and repeated light stimulation enhances the response of visual neurons by increasing sodium currents in Xenopus tadpoles (Aizenman et al., 2003). Our study adds to the literature describing unexpected excitability changes following altered sodium conductance.

\section{Correlations between maximal conductance measurements}

Previous work has shown that ion channel mRNA levels may covary (Schulz et al., 2006, 2007) and that conductances are not regulated in isolation (Desai et al., 1999; O'Leary et al., 2010). Instead, individual conductances may be tightly correlated with other conductances, sometimes with those that have opposite functions, suggesting that neurons use multiple mechanisms to reach a homeostatic set point. The models generated for this study had conductance values consistent with the generation of tonic firing. When we plotted the gain of each model versus its baseline sodium conductance, we found no linear relationship between these quantities (data not shown), suggesting that gain is not inherently higher with more $\mathrm{Na}^{+}$conductance for models in the population. The gain changes observed in this study occurred only after the baseline models were perturbed, effectively altering the balance between the conductances.

We additionally considered models with multiple conductance changes similar to those seen in experimental work in activity-deprived neurons in which $\mathrm{Na}^{+}$and $K_{\mathrm{d}}$ conductances change in concert (Desai et al., 1999). In those experiments, sodium conductance increased by a factor of $1.3 \times$ and $K_{\mathrm{d}}$ conduc- 
tance decreased by $0.6 \times$ after chronic tetrodotoxin blockade. Both sodium and $K_{\mathrm{d}}$ conductances changes of that magnitude independently decreased the gain at high firing rates. When combined in the model, they produced $\sim 50 \%$ of the full effect we report for a $3 \times$ sodium conductance increase (data not shown). It is possible that if a dramatic change to the $\mathrm{Na}^{+}$conductance occurred in a real nervous system due to a genetic alteration, for example, other conductances might compensate (Alkon, 1984; Mee et al., 2004; Swensen and Bean, 2005; Laezza et al., 2007; Lorenzetto et al., 2009). Even though such a process is not explicitly possible in our model, the voltage-dependent, and thus inexorably linked, conductances compensated for one another regardless of this. As we have shown, the enhancement of the $\mathrm{Na}^{+}$conductance results in an enhancement of the $K_{\mathrm{d}}$ conductance that was strong enough to lower the firing rate overall. Thus, the opposing nature of the $\mathrm{Na}^{+}$and $K_{\mathrm{d}}$ conductances, and their joint dependence on the voltage, causes them to provide a rudimentary mechanism for homeostatic control in response to a single perturbation, even though we did not explicitly endow the model with a mechanism to achieve this.

The reduced models presented in our study did not simulate calcium entry during action potentials. The increases in sodium conductance resulted in wider action potentials which would likely lead to increased calcium entry in real neurons. Calcium is well known to trigger a variety of intracellular processes which could influence the input-output relationship of real neurons or trigger additional homeostatic feedback mechanisms in ways not addressed by these simulation results.

\section{Reliable neuromodulation in a simple model}

An interesting question that arises from observing variability in nervous systems is that it can seem unclear how neuromodulators can have reliable effects on their targets if those targets are highly heterogeneous (Marder and Thirumalai, 2002). In practice, external stimuli such as therapeutic drugs affect the majority of a population in a similar manner even though it is self-evident that individuals in that population must have brain circuits constructed with different numbers of neurons, different synaptic weights and different histories of neuromodulation and activation. Previous work has investigated this phenomenon experimentally (Grashow et al., 2009). Theoretical work has shown how a model neuron with a diverse parameter space can be robust to change but still respond reliably to neuromodulation (Goldman et al., 2001) and investigated the structure of the parameter space of heterogeneous models with similar behavior (Achard and De Schutter, 2006). Our study fits into this conceptual framework by demonstrating a highly consistent FI curve change in a population of heterogeneous models in response to the same conductance density change.

\section{Implications for homeostatic control mechanisms}

Commonly, work describing neuronal homeostasis has made the implicit assumption that the mean firing rate of a neuron is the quantity that should be controlled (Turrigiano et al., 1995, 1998). However, it is well appreciated that neurons can have a large range of firing rates and a key property of neuronal computation is that neurons can meaningfully respond to a wide variety of input levels. This requires a homeostatic feedback process that not only ensures that mean firing rate remains at a reasonable level but instead ensures that the entire input-output relationship is kept in a computationally meaningful range. Our study demonstrates that changing intrinsic conductances can sometimes lead to anti-homeostatic effects on the input-output relationship.

\section{References}

Abbott LF, Varela JA, Sen K, Nelson SB (1997) Synaptic depression and cortical gain control. Science 275:220-224.

Achard P, De Schutter E (2006) Complex parameter landscape for a complex neuron model. PLoS Comput Biol 2:e94.

Aizenman CD, Akerman CJ, Jensen KR, Cline HT (2003) Visually driven regulation of intrinsic neuronal excitability improves stimulus detection in vivo. Neuron 39:831-842.

Alkon DL (1984) Calcium-mediated reduction of ionic currents: a biophysical memory trace. Science 226:1037-1045.

Cantrell AR, Catterall WA (2001) Neuromodulation of $\mathrm{Na}^{+}$channels: an unexpected form of cellular plasticity. Nat Rev Neurosci 2:397-407.

Carter BC, Bean BP (2011) Incomplete inactivation and rapid recovery of voltage-dependent sodium channels during high-frequency firing in cerebellar Purkinje neurons. J Neurophysiol 105:860-871.

Chance FS, Abbott L, Reyes AD (2002) Gain modulation from background synaptic input. Neuron 35:773-782.

Connor JA, Stevens CF (1971) Prediction of repetitive firing behaviour from voltage clamp data on an isolated neurone soma. J Physiol 213:31-53.

Connor JA, Walter D, McKown R (1977) Neural repetitive firing: modifications of the Hodgkin-Huxley axon suggested by experimental results from crustacean axons. Biophys J 18:81-102.

Dayan P, Abbott LF (2001) Theoretical neuroscience: computational and mathematical modeling of neural systems, Ed 1. Cambridge, MA: MIT.

Desai NS, Rutherford LC, Turrigiano GG (1999) Plasticity in the intrinsic excitability of cortical pyramidal neurons. Nat Neurosci 2:515-520.

Destexhe A, Paré D (1999) Impact of network activity on the integrative properties of neocortical pyramidal neurons in vivo. J Neurophysiol 81:1531-1547.

Destexhe A, Rudolph M, Paré D (2003) The high-conductance state of neocortical neurons in vivo. Nat Rev Neurosci 4:739-751.

Fernandez FR, Mehaffey WH, Turner RW (2005) Dendritic $\mathrm{Na}^{+}$current inactivation can increase cell excitability by delaying a somatic depolarizing afterpotential. J Neurophysiol 94:3836-3848.

Gabbiani F, Krapp HG, Koch C, Laurent G (2002) Multiplicative computation in a visual neuron sensitive to looming. Nature 420:320-324.

Goaillard JM, Taylor AL, Schulz DJ, Marder E (2009) Functional consequences of animal-to-animal variation in circuit parameters. Nat Neurosci 12:1424-1430.

Goldman MS, Golowasch J, Marder E, Abbott LF (2001) Global structure, robustness, and modulation of neuronal models. J Neurosci 21:5229-5238.

Golowasch J, Abbott LF, Marder E (1999) Activity-dependent regulation of potassium currents in an identified neuron of the stomatogastric ganglion of the crab Cancer borealis. J Neurosci 19:RC33-RC33.

Grashow R, Brookings T, Marder E (2009) Reliable neuromodulation from circuits with variable underlying structure. Proc Natl Acad Sci U S A 106:11742-11746.

Günay C, Edgerton JR, Jaeger D (2008) Channel density distributions explain spiking variability in the globus pallidus: a combined physiology and computer simulation database approach. J Neurosci 28:7476-7491.

Harvey CD, Collman F, Dombeck DA, Tank DW (2009) Intracellular dynamics of hippocampal place cells during virtual navigation. Nature 461:941-946.

Hodgkin A (1975) The optimum density of sodium channels in an unmyelinated nerve. Philos Trans R Soc Lond B Biol Sci 270:297-300.

Hodgkin A, Huxley A (1952) A quantitative description of membrane current and its application to conduction and excitation in nerve. J Physiol 117:500-544.

Holt GR, Koch C (1997) Shunting inhibition does not have a divisive effect on firing rates. Neural Comput 9:1001-1013.

Kim J, Tsien RW (2008) Synapse-specific adaptations to inactivity in hippocampal circuits achieve homeostatic gain control while dampening network reverberation. Neuron 58:925-937.

Laezza F, Gerber BR, Lou JY, Kozel MA, Hartman H, Craig AM, Ornitz DM, Nerbonne JM (2007) The FGF14(F145S) mutation disrupts the interaction of FGF14 with voltage-gated $\mathrm{Na}+$ channels and impairs neuronal excitability. J Neurosci 27:12033-12044. 
Liu Z, Golowasch J, Marder E, Abbott LF (1998) A model neuron with activity-dependent conductances regulated by multiple calcium sensors. J Neurosci 18:2309-2320.

Lorenzetto E, Caselli L, Feng G, Yuan W, Nerbonne JM, Sanes JR, Buffelli M (2009) Genetic perturbation of postsynaptic activity regulates synapse elimination in developing cerebellum. Proc Natl Acad Sci U S A 106:16475-16480.

Lundstrom BN, Hong S, Higgs MH, Fairhall AL (2008) Two computational regimes of a single-compartment neuron separated by a planar boundary in conductance space. Neural Comput 20:1239-1260.

Lundstrom BN, Famulare M, Sorensen LB, Spain WJ, Fairhall AL (2009) Sensitivity of firing rate to input fluctuations depends on time scale separation between fast and slow variables in single neurons. J Comput Neurosci 27:277-290.

Marder E (2011) Colloquium paper: variability, compensation, and modulation in neurons and circuits. Proc Natl Acad Sci USA 108: 15542-15548.

Marder E, Bucher D (2007) Understanding circuit dynamics using the stomatogastric nervous system of lobsters and crabs. Annu Rev Physiol 69:291-316.

Marder E, Taylor AL (2011) Multiple models to capture the variability in biological neurons and networks. Nat Neurosci 14:133-138.

Marder E, Thirumalai V (2002) Cellular, synaptic and network effects of neuromodulation. Neural Netw 15:479-493.

Mee CJ, Pym ECG, Moffat KG, Baines RA (2004) Regulation of neuronal excitability through pumilio-dependent control of a sodium channel gene. J Neurosci 24:8695-8703.

Mitchell SJ, Silver RA (2003) Shunting inhibition modulates neuronal gain during synaptic excitation. Neuron 38:433-445.

Moyer JR Jr, Thompson LT, Disterhoft JF (1996) Trace eyeblink conditioning increases $\mathrm{CA} 1$ excitability in a transient and learning-specific manner. J Neurosci 16:5536-5546.

Murayama M, Pérez-Garci E, Nevian T, Bock T, Senn W, Larkum ME (2009) Dendritic encoding of sensory stimuli controlled by deep cortical interneurons. Nature 457:1137-1141.

O’Leary T, van Rossum MCW, Wyllie DJA (2010) Homeostasis of intrinsic excitability in hippocampal neurones: dynamics and mechanism of the response to chronic depolarization. J Physiol 588:157-170.

Paré D, Shink E, Gaudreau H, Destexhe A, Lang EJ (1998) Impact of spontaneous synaptic activity on the resting properties of cat neocortical pyramidal neurons in vivo. J Neurophysiol 79:1450-1460.

Prescott SA, De Koninck Y (2003) Gain control of firing rate by shunting inhibition: roles of synaptic noise and dendritic saturation. Proc Natl Acad Sci U S A 100:2076-2081.

Prescott SA, Ratté S, De Koninck Y, Sejnowski TJ (2006) Nonlinear interaction between shunting and adaptation controls a switch between integration and coincidence detection in pyramidal neurons. J Neurosci 26:9084-9097.
Prinz AA, Bucher D, Marder E (2004) Similar network activity from disparate circuit parameters. Nat Neurosci 7:1345-1352.

Rosbash M (2009) The implications of multiple circadian clock origins. PLoS Biol 7:e62.

Rothman JS, Cathala L, Steuber V, Silver RA (2009) Synaptic depression enables neuronal gain control. Nature 457:1015-1018.

Salinas E, Thier P (2000) Gain modulation: a major computational principle of the central nervous system. Neuron 27:15-21.

Schulz DJ (2006) Plasticity and stability in neuronal output via changes in intrinsic excitability: it's what's inside that counts. J Exp Biol 209: $4821-4827$.

Schulz DJ, Goaillard JM, Marder E (2006) Variable channel expression in identified single and electrically coupled neurons in different animals. Nat Neurosci 9:356-362.

Schulz DJ, Goaillard JM, Marder EE (2007) Quantitative expression profiling of identified neurons reveals cell-specific constraints on highly variable levels of gene expression. Proc Natl Acad Sci U S A 104:13187-13191.

Silver RA (2010) Neuronal arithmetic. Nat Rev Neurosci 11:474-489.

Stemmler M, Koch C (1999) How voltage-dependent conductances can adapt to maximize the information encoded by neuronal firing rate. Nat Neurosci 2:521-527.

Swensen AM, Bean BP (2005) Robustness of burst firing in dissociated Purkinje neurons with acute or long-term reductions in sodium conductance. J Neurosci 25:3509-3520.

Tang LS, Goeritz ML, Caplan JS, Taylor AL, Fisek M, Marder E (2010) Precise temperature compensation of phase in a rhythmic motor pattern. PLoS Biol 8:e1000469.

Tang LS, Taylor AL, Rinberg A, Marder E (2012) Robustness of a rhythmic circuit to short- and long-term temperature changes. J Neurosci 32: 10075-10085.

Taylor AL, Goaillard JM, Marder E (2009) How multiple conductances determine electrophysiological properties in a multicompartment model. J Neurosci 29:5573-5586.

Triesch J (2007) Synergies between intrinsic and synaptic plasticity mechanisms. Neural Comput 19:885-909.

Turrigiano G, LeMasson G, Marder E (1995) Selective regulation of current densities underlies spontaneous changes in the activity of cultured neurons. J Neurosci 15:3640-3652.

Turrigiano GG, Leslie KR, Desai NS, Rutherford LC, Nelson SB (1998) Activity-dependent scaling of quantal amplitude in neocortical neurons. Nature 391:892-896.

Wark B, Lundstrom BN, Fairhall A (2007) Sensory adaptation. Curr Opin Neurobiol 17:423-429.

Zhang W, Linden DJ (2003) The other side of the engram: experiencedriven changes in neuronal intrinsic excitability. Nat Rev Neurosci 4:885900 . 